\title{
Final bulletin
}

\section{L LEIKER, A M DHOPLE \& ENNO FREERKSEN}

In the sixties it became obvious that the era of successful monotherapy with dapsone would soon come to an end, due to worldwide increasing emergence of sulphone resistance of Mycobacterium leprae. Since then the innovating concepts of Professor Freerksen have become the basis for a new approach in the treatment of leprosy. Based on his experience in tuberculosis he called for multidrug therapy in leprosy as in tuberculosis with therapy of limited duration, for shorter periods than in the sulphone era. He took into account the element of human weakness in taking drugs and introduced the first combined formula with all drugs in a single tablet, undoubtedly a realistic measure to prevent the intake of only one drug by a patient, which would lead to resistance to this drug.

For the first time detailed information, including independent assessments of the Malta Leprosy Eradication Project, were made public. The results, after a follow-up period of 10 years after release from treatment, are by all standards excellent.

Although the drug combination proposed by Professor Freerksen are not accepted by everyone, his principles for chemotherapy are now generally accepted. The Malta experiences formed the basis for the recommendations in 1982 by WHO Study Group on Chemotherapy of chemotherapy.

It is now generally accepted that MDT is the only answer to the emergence of drug resistance. We may expect a higher rate of cure after a shorter treatment period than before. If MDT is properly applied we may expect that it will bring the control of leprosy a major step nearer.

We have listened to reports on the use of rifampicin-Isoprodian in a leprosy and TB control project in Paraguay; the results were favourable. We have listened to reports on experiences with the WHO Study Group regimen from India, Malawii, Nepal, Ethiopia, Tanzania, Sierra Leone and the Dominican Republic. These reports show that in spite of many difficulties which must be overcome, MDT is operationally feasible also in situations where the infrastructure is less favourable than in Malta. The great challenge is now to apply MDT worldwide. close cooperation between governments and voluntary agencies specializing in 
leprosy aid, such as the German Leprosy Relief Association, will be a necessity to reach this goal.

A randomized multicentre study did not reveal measurable differences between a dapsone monotherapy regimen, a combination of dapsone and rifampicin and rifampicin plus Isoprodian, but this applies only to the treatment phase. Time must teach what will happen hereafter.

In Turkey a short course MDT regimen of only 6 months resulted in no relapses in $29 \mathrm{MB}$ patients af ter a follow-up period of 2-3 years.

In the Netherlands about 400 patients, one third MB patients, were treated for 1 year with daily rif ampicin, dapsone and clofazimine on alternate days. During a follow-up period of 3-5 years only one relapse was seen, the results are not significantly different from those in Malta.

In Malta morphologically intact bacilli were found in some patients, without clinical evidence of relapse. In Paraguay few relapses were recorded. This suggests that none of the present regimens is capable of eliminating all persisters. However, precise information about the significance of small numbers of persisters for the risk of relapse is still lacking.

The conference shows that although we are now beginning to see clearly the direction in which we have to go for controlling leprosy, we still have much to learn about the exact way.

Little is still known about the minimum duration of treatment required for obtaining acceptably low relapse rates with the different regimens.

The reasons for finding little toxicity af ter regimens with PTH in several areas and significant toxicity in others is yet not clarified.

Seemingly simple questions such as reliable criteria for distinguishing between relapse and reversal reaction which are essential for assessing relapse rates, are not yet available.

During discussions a strong point was made of the need for standardizations of definitions. We must learn from all MDT projects. Each project is in fact a trial. But we can learn only when authors state in their reports their meaning of definitions used.

Earlier I had said that we can expect much from MDT if it is properly applied with the emphasis on properly. But will this really happen? I doubt it very much. Drugs are available on the black market or without a prescription in pharmacies. Drug abuse cannot be entirely avoided.

Therefore in the long run there remains the threat of emergence of drug resistance, in particular to rifampicin. This brings me to the second part of the conference.

In addition to making use of the presently available tools in the best possible way there is an urgent need for development of new tools. Strengthening a fundamental research deserves a high priority. Since research is time-consuming and it takes many years before the fruits of research have been tested and can be applied in the control of the disease we cannot afford delays. 


\section{THE SECOND HALF OF THE CONFERENCE DEALT WiTh THESE SUBJECTS}

The inability to cultivate $M$. leprae, the causative agent of human leprosy, has been a bottle-neck in leprosy research since its discovery in 1872 by Armauer Hansen. However, some significant progress was reported during this Symposium. Along with microscopic counting of acid-fast bacteria in cultures, various biochemical parameters were used to evaluate the in vitro growth of $M$. leprae. The primary isolates retained all the characteristic of $M$. leprae even though subcultures could not be achieved. The old theory of $M$. leprae being a 'microbe-dependent microbe' was again brought to the surface.

Environmentally derived mycobacteria were isolated from soil samples collected from leprosy endemic areas and were identified as resembling M. leprae. This was confirmed by inoculating into footpads of normal and nude mice. However, it was suggested to differentiate soil-derived cultivable mycobacteria from M. leprae.

There is an urgent need to develop newer antileprosy compounds for use in endemic areas. It was suggested to employ metabolic activities in $M$. leprae, which are essential for growth and survival of bacteria, as possible targets of antileprosy agents. Such targets can be cell wall synthesis and proteins synthesis. Nucleic acid synthesis seems to be another target and two known antileprosy agents, rif ampicin and clofazimine, appear to affect these pathways.

The most significant contribution of this Symposium was the presentations on in vitro test systems for rapid screening of potential antileprosy compounds. One method was the in vitro culture system where biochemical parameters were used to evaluate the effects of drugs on $M$. leprae. Another was the use of hypoxanthine incorporation by $M$. leprae. In other test systems $M$. leprae were phygocytosed by human and mice macrophages and the effects of drugs on $M$. leprae were evaluated inside these macrophages using various test systems. These systems include $\mathrm{Fe}$ receptor assay using EA rosetting technique, fluorescence staining using fluorecin diacetate, uracil uptake assay and sialic acid assay. In all these test systems effects of known antileprosy compounds were tested to offer assurance about the feasibility of these systems and encouraging results on the efficacy of some newer compounds on the viability of $M$. leprae were presented. High technology instrumentation has also been employed to assess the viability of $M$. leprae. This dealt with the use of mass spectrometric analysis of single M. leprae cells obtained from the biopsies of patients under chemotherapy. The information is derived from the intracellular concentration of sodium and potassium ions and also from fragment ions of the complex cell matrix. Excellent correlation was described between the results obtained with this technique and those obtained by ATP assay and mouse footpad assay.

The current feeling is that leprologists will accept these systems provided it can be shown that these results are parallel to those obtained by the mouse footpad model. So the mouse footpad system was described in full detail and 
explanations on continuous method, kinetic method and proportional bactericidal method were offered. It was further reported that the kinetic method might be most suitable since this method distinguishes bactericidal drugs from bacteriostatic drugs. With this method several new classes of compounds have been tested. It was also reported that drug monitoring trials can be undertaken utilizing immunologically suppressed mice and rats including nude rats.

It seems the laboratory investigators are moving in a proper direction and that such studies should receive constant encouragement from all concerned with leprosy.

The new routes for the development and screening of potential antileprotic drugs and drug combinations. The new compounds were synthesized with certain enzymes of bacteria as targets. The best example being the dihydrofolate reductase of both $M$. lufu and $M$. leprae. Both whole cells and cell-free enzymes were used. Several trimethoprim derivatives were synthesized and two compounds namly K-130 and Brodimoprim were found to be effective against both the sytems of both bacteria. Furthermore, synergistic activities were also observed with these compounds. Another target enzyme used was ribonucleotide reductase to evaluate the effects of thiosemicarbozones. 